\title{
p53 regulates epithelial-mesenchymal transition and stem cell properties through modulating miRNAs
}

\author{
Chun-Ju Chang, Chi-Hong Chao, Weiya Xia, Jer-Yen Yang, Yan Xiong, Chia-Wei Li, Wen-Hsuan Yu, Sumaiyah K. \\ Rehman, Jennifer L. Hsu, Heng-Huan Lee, Mo Liu, Chun-Te Chen, Dihua Yu and Mien-Chie Hung
}

Nat. Cell Biol. 13, 317-323 (2011); published online 20 February 2011

In the version of this article initially published online and in print, Figs $2 \mathrm{~b}$ and $4 \mathrm{a}$ contained panels that did not match those in Supplementary Fig. S5, which showed full uncropped scans corresponding to the specific figures. In Fig. $2 b$, the p53 panel was stretched so that the image was thinner than that shown in Supplementary Fig. S5. The p53 image was inadvertently duplicated and shown in place of the correct E-cadherin panel. The $\mathrm{N}$-cadherin panel was derived from an independent experiment, and not the scan shown in Supplementary Fig S5. Finally, the actin panel was stretched and reversed, compared with Supplementary Fig. S5. The correct images are shown below. In the image shown below, the N-cadherin panel now corresponds to the scan shown in Supplementary Fig. S5.

In Fig. 4a (right), the BMI1 panel was stretched vertically, compared with Supplementary Fig. S5. The panel for actin was incorrect in both Fig. 4a (right) and Supplementary Fig. S5. As shown below in the amended figure for Supplementary Fig. S5, samples from two experiments, relating to Fig. 4a and to Supplementary Fig. S1d, were run alongside each other. When preparing images for Fig. 4a of the original manuscript, the image of the gel was reversed, and when cropped the incorrect bands were then shown in Fig. 4a and Supplementary Fig. S5 (the correct image was shown in Supplementary Fig. S1d, however). The amended image for Fig. 4a and Supplementary Fig. S5 are shown below. The red line in the uncropped scan shown below indicates where the scan was cropped; lanes 1-5 are now shown in Fig. 4a, and lanes 6-8 were shown in Supplementary Fig. S1d in the original manuscript.

We apologize for these serious mistakes. The original conclusions of the paper are unchanged.

Figure 2

b

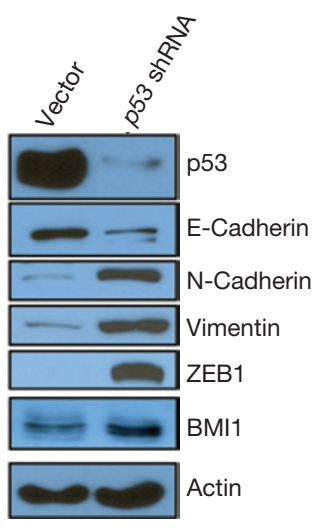

Figure 4

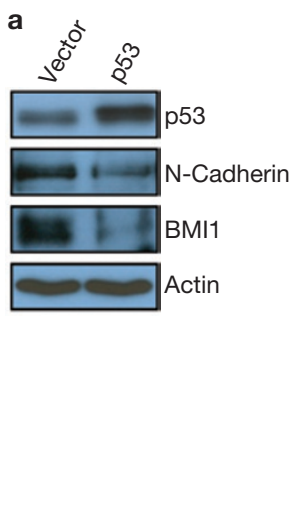

Figure S5

Corresponding to Figure $4 a$

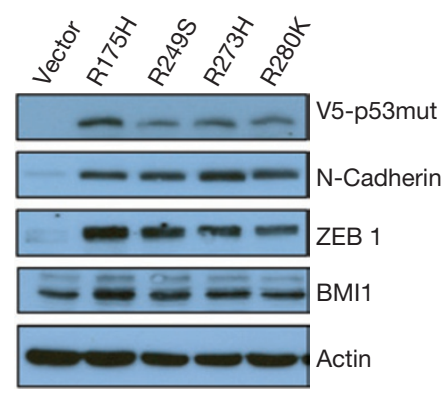

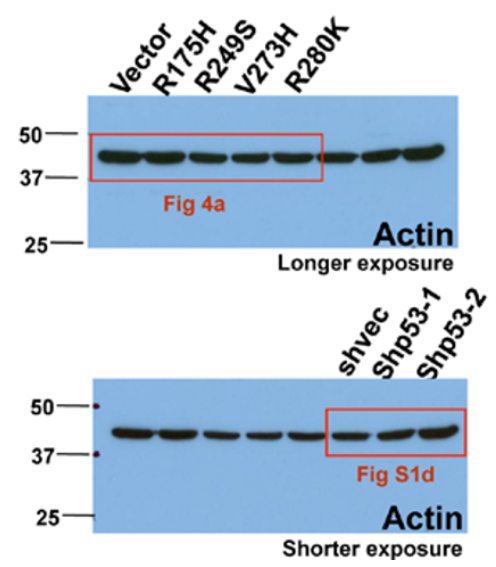

